\title{
Intervention par plongeurs sur les installations d'une pile piscine (Osiris - G.E.N. Saclay)
}

\author{
LA PRÉVENTION TECHNIQUE ET MÉDICALE : \\ UN EXEMPLE DE DÉMARCHE ERGONOMIQUE \\ MULTIDISCIPLINAIRE
}

\author{
C. ARNOULD $(*)$ et L. MARTIN $(* *)$
}

(Manuscrit reçu le 2 février 1979)

\begin{abstract}
RÉSUMÉ
Des travaux de maintenance sur une pile piscine ont été réalisés par une équipe de plongeurs. Une étude ergonomique multidisciplinaire a permis de définir la méthode de travail. La démarche ergonomique est analysée. La technique de travail des plongeurs est décrite. Le bilan médical montre que le chantier confirme les prévisions et que l'on dispose d'une méthode sûre et éprouvée. Cette technique d'intervention humaine directe, sous l'eau, pourrait ouvrir de nouvelles possibilités d'action en industrie nucléaire.
\end{abstract}

\begin{abstract}
Maintenance works in a swimming-pool reactor was performed by a team of divers. A multidisciplinary ergonomic study had previously defined the working procedure. The ergonomic approach is analysed. The divers' working techniques are described. After work, medical tests showed that previsions were verified and proved the methods as safe. This technique by divers' interventions should open new possibilities in nuclear industry.
\end{abstract}

\section{PRÉAMBULE}

Des travaux à première vue difficilement réalisables par intervention humaine directe, ont été effectués par des plongeurs sur les installations d'une pile piscine. Une analyse détaillée, multidisciplinaire, de l'objectif et

(*) Médecin du travail, S.M.T./Essais, C.E.A./Direction Essais, Centre d'Études de Bruyères-le-Châtel, B.P. $n^{\circ}$ 561, 92542 Montrouge Cedex.

$\left(^{* *}\right)$ Chef plongeur, S.P.S., C.E.A./Direction Essais, B.P. $\mathrm{n}^{\circ}$ 17, 91310 Montlhéry.

RADIOPROTECTION, vOL. 14 - 0033-8451/1979/67/\$ 4.00/C Bordas-Dunod. 
des conditions de travail a permis de préparer et d'organiser le chantier sans imposer de contraintes inhabituelles aux plongeurs.

Nous exposerons les données générales du problème, le développement de l'étude préalable de prévention technique et médicale, les constatations faites sur le chantier et notamment le vécu des travaux par les participants, principalement par les plongeurs eux-mêmes. Nous terminerons par un examen critique de l'étude ergonomique préalable à la lumière des données relevées effectivement sur le terrain, pendant les travaux. Nous conclurons en soulignant l'intérêt de l'approche multidisciplinaire dans une telle démarche ergonomique et les avantages que l'on a pu en retirer sous l'angle de la prévention. La coopération étroite entre chef des plongeurs et médecin du travail a permis notamment de résoudre de façon simple des problèmes de chantier complexes a priori, en appliquant des méthodes de travail connues et éprouvées.

\section{LE PROBLÈME TECHNIQUE}

\section{LA « FAISABILITÉ »}

Pour l'exploitant de la pile piscine Osiris, installation nucléaire du C.E.N.-Saclay, il s'agit de faire réaliser certaines opérations de contrôle et de maintenance sur les capacités en eau de la pile, notamment au niveau des bacs de refroidissement cœur et piscine. La solution classique, à sec, après vidange de l'installation suppose la mise en œuvre d'une stratégie complexe, comportant obligatoirement d'assez longs travaux d'assainissement et de contrôles radiologiques et, par conséquent, une immobilisation prolongée de la pile (de l'ordre de 1 an).

L'intervention directe, par plongeurs, est envisagée en raison du peu de perturbations qu'elle est susceptible d'entraîner sur l'installation : la pile est arrêtée, mais il n'est pas nécessaire de vidanger pour effectuer les travaux. Le temps d'immobilisation de la pile est donc très réduit (de l'ordre de 1 à 2 mois), les travaux de radioprotection sont très simplifiés. En première analyse :

- le C.E.A./Essais dispose d'un groupe de plongeurs professionnels compétents pour des travaux sous-marins en ambiance nucléaire et a priori parfaitement capables de prendre en charge ce type de chantier;

- la contamination de l'eau est très limitée du fait de son passage sur résines. De plus, l'utilisation de vêtements de plongée isolants permet de s'en prémunir;

- le problème particulier de la contamination par eau tritiée peut être facilement calculé par des mesures préalables sur l'eau des capacités. Les doses intégrées à prévoir sont très faibles, pratiquement négligeables;

- l'enlèvement par les plongeurs d'éventuels dépôts actifs doit être grandement facilité par la protection apportée par l'eau vis-à-vis des rayonnements gamma; 
- la température de l'eau peut être ramenée à un niveau confortable pour les plongeurs;

- la géométrie des volumes à visiter permet l'accessibilité par les plongeurs. Les matériels nécessaires aux travaux peuvent être choisis en fonction de leur encombrement et de leur maniabilité.

La sécurité des plongées en espace clos n'est pas péjorative $\left({ }^{1}\right)$ dans la mesure où il est fait appel à des plongeurs professionnels. Les moyens de liaison avec la surface, les moyens d'intervention rapide peuvent être mis en place. La surveillance spéciale de plongeurs travaillant en milieu actif est un problème bien connu du médecin du travail du groupe plongeurs. Cette première approche de "faisabilité » apparaît donc positive.

\section{L'ÉTUDE DU POSTE DE TRAVAIL AU NIVEAU DE LA CONCEPTION}

\subsection{REgROUPEMENT ET ANALYSE DES DONNÉES PAR DISCIPLINE}

L'analyse détaillée du poste de travail est entreprise dans le but de faire apparaître, avec le plus de précision possible, discipline par discipline, les différents paramètres du futur poste de travail. Une telle étude préliminaire (classique d'ailleurs pour les travaux spécialisés en milieu hostile) permet d'intégrer dès la conception les contraintes humaines et techniques, et de définir les mesures et moyens de sécurité qui seront mis en place sur le terrain.

\section{Les données techniques: l'exploitant de la pile}

L'objectif se définit ainsi :

- visiter un certain nombre de points des capacités en eau de la pile : bac cœur, bac piscine, canaux, piscine elle-même;

- procéder à l'enlèvement de dépôts actifs se trouvant éventuellement dans des points non directement accessibles en conditions de fonctionnement normal (bacs de refroidissement, par exemple). Ce point sera décidé sur place au moment de l'inspection par les plongeurs;

- pratiquer une inspection méthodique des parois, des canalisations et des vannes accessibles, par contrôle visuel, par photographie et par télévision : (les images télévisées seront retransmises en surface et enregistrées sur magnétoscope);

- effectuer à la demande, au vu du bilan fait par les plongeurs, certains travaux de maintenance (raccords d'enduits, intervention sur les canalisations, etc.) et certains travaux de décontamination éventuels (brossage, aspiration).

(1) La prise de risque est la même que pour certains chantiers sous-marins traditionnels (travaux dans des canalisations, par exemple).

voL. $14-\mathrm{N}^{\circ} 2$ 
Les contraintes techniques, liées à la pile elle-même, sont examinées par rapport à l'objectif :

Le plan des installations fait l'objet d'un examen particulièrement attentif par l'exploitant et par le chef des plongeurs, notamment pour les bacs de refroidissement cœur et piscine. La sécurité des accès, les volumes, leur géométrie, sont étudiés en fonction des possibilités d'évolution des plongeurs et de leur sécurité. Il s'agit d'espaces restreints, clos, sans possibilité de remontée rapide en surface en cas d'incident, problème de sécurité dont les plongeurs tiennent compte dans l'organisation de leur chantier.

La température de l'eau, qui trop élevée pourrait entraîner une fatigue importante pour les plongeurs, sera ramenée à un niveau confortable (28 à $30^{\circ} \mathrm{C}$ ) par action sur les circuits eau de la pile.

La sécurité hydraulique du chantier de plongée doit être assurée, puisque le niveau dans la pile doit rester supérieur au niveau de remplissage des points de plongée. L'isolement est assuré par la mise en place d'un batardeau équipé de dispositifs de surveillance et d'alerte à distance.

\section{Données radiologiques : le Service de protection radiologique}

Pour préciser l'environnement radiologique auquel seront soumis les plongeurs, deux points sont à considérer :

Le problème de l'irradiation, d'une part, se présente de la façon suivante : le réacteur est stoppé, le cœur est stocké sous eau dans un canal de la pile. Les sources susceptibles de provoquer une irradiation dans les lieux de plongée. " bac cœur » en particulier, sont évacuées et placées à une distance suffisante pour que l'irradiation provoquée sur les points de plongée soit négligeable. Cette action est menée par l'exploitant de la pile sur avis du Service de protection contre les rayonnements (S.P.R.).

Il subsiste le problème de l'irradiation due à la présence éventuelle de matériaux activés dans le cœur et décantés dans les bacs. Aucun élément ne permet de préciser a priori ni le volume ni l'activité de tels matériaux au niveau des bacs cœur et piscine. Le doute sera levé sur place au moment de la reconnaissance radiologique des lieux, première plongée de reconnaissance avec radiamètre portatif. Cette procédure est la même qu'en radioprotection de surface avec, cependant, un gros avantage pour le plongeur qui bénéficie de la protection apportée par l'eau vis-à-vis des rayonnements gamma. Au cours de cette première phase, le risque d'irradiation est apprécié à partir des relevés de mesures d'irradiation (D.G.E.T.) sur les parois et les sources métalliques éventuelles.

Le problème contamination, d'autre part, tient compte des éléments suivants :

le risque de contamination de l'eau par des éléments en suspension, ou déposés sur les parois, est faible en raison de l'épuration de l'eau des capacités par passage sur résines. Il est apprécié à partir des mesures sur les prélèvements d'eau avant les travaux et au cours des interventions plongeurs pour évaluer le facteur de remise en suspension. De plus, les plongeurs sont protégés de ce type de contamination par le port de vêtements isolants; 
la contamination du circuit par l'eau tritiée constitue un risque à part en raison de sa très grande diffusibilité. Les mesures préalables effectuées par le S.P.R. sur l'eau du circuit donnent une activité volumique due au tritium de $1,5 \cdot 10^{-2} \mathrm{Ci} / \mathrm{m}^{3}$ correspondant pour la durée d'une période de travail à un équivalent de dose engagée inférieur à $10 \mathrm{mrem}$, ce qui est négligeable.

Dans ces conditions, on peut admettre que l'environnement radiologique dans lequel évolueront les plongeurs n'entraîne pas de contraintes autres que celles de tous travaux en zone contrôlée : surveillance de l'ambiance, dosimétrie individuelle, contrôles radiologiques en fin de plongée, surveillance médicale spéciale systématique.

Il est à noter que les plongeurs choisis pour ces travaux sont tous des techniciens de radioprotection confirmés.

\section{L'organisation pratique des travaux. Le chef du groupe de plongeurs}

Connaissant parfaitement la mission et les données concernant l'environnement, le chef du groupe de plongeurs peut planifier son chantier et définir les moyens nécessaires.

\section{distinctes :}

Le déroulement des travaux. - Il se décompose logiquement en séquences

ouverture des accès et reconnaissance radiologique; assainissement radiologique; visite réglée des capacités : prises de vue photographiques et télévisées; travaux de maintenance.

Cette chronologie n'appelle aucune remarque sur le plan ergonomique.

Les moyens nécessaires (fig. 1 et 2) :

- en personnels : équipes de deux plongeurs en rotation, deux ou trois équipes de deux, 2 heures de travaux en plongée par plongeur et par jour (durée des travaux de l'ordre de 3 semaines);

- en matériels : nous passerons rapidement sur les matériels techniques, outils de travail nécessaires aux travaux proprement dits; ils ne présentent aucune particularité sinon d'être choisis ou adaptés en fonction du problème de l'encombrement en espace restreint (caméra, télévision, projecteurs, suceuse, etc.).

L'équipement individuel des plongeurs mérite, par contre, quelques précisions. Cet équipement est très classique en travaux sous-marins. Les vêtements et les détendeurs ont été simplement adaptés pour renforcer leur étanchéité.

Les vêtements utilisés sont des tenues isolantes Draeger, du type à " volume constant ", en caoutchouc souple avec ajustage de la cagoule sur l'habit au niveau du cou. L'embout respiratoire est solidaire de la cagoule. L'équilibrage du vêtement se fait par une soupape de tête située au niveau de la cagoule et une soupape au niveau de chaque pied, ceci pour éviter le phénomène de "ballon ». Les soupapes sont montées de façon à éviter les entrées d'eau (prévention du risque de contamination). Sous cet habit, les 


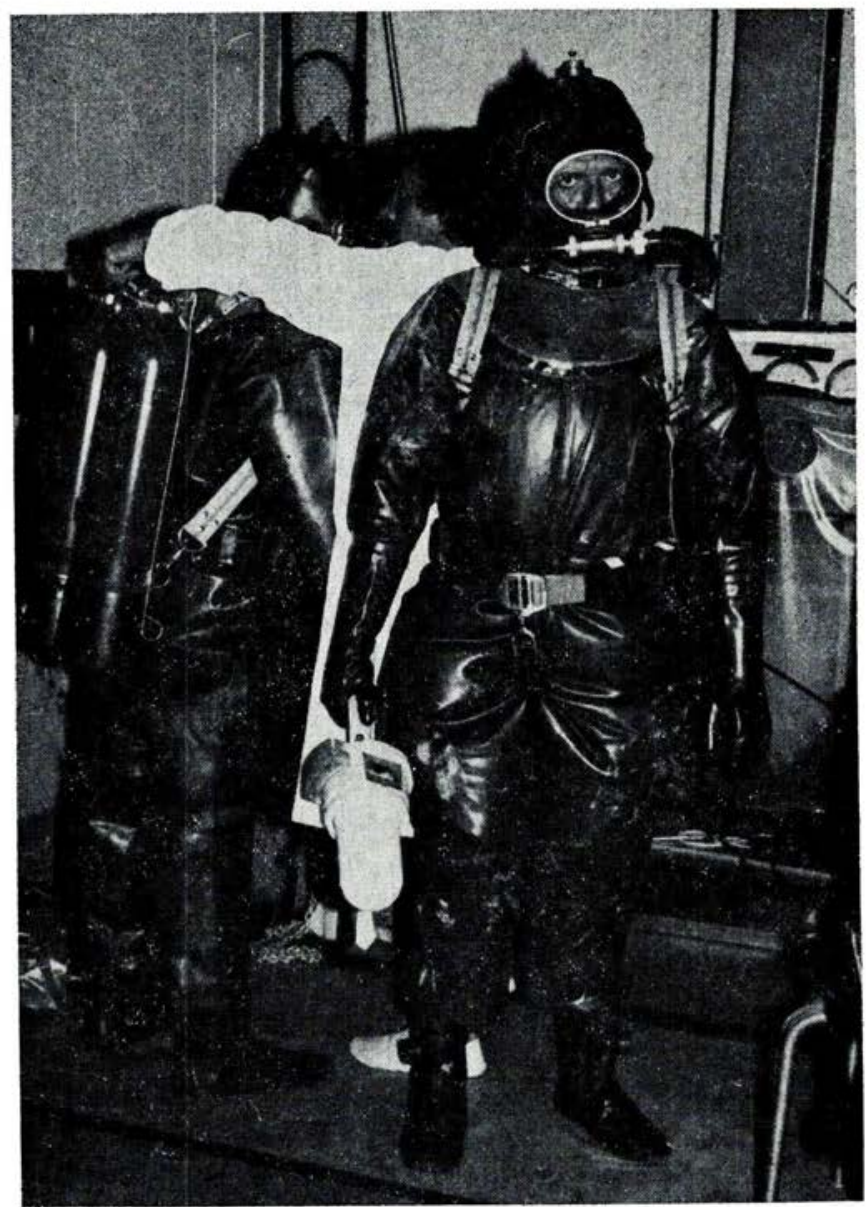

FIG. 1. - Plongeur muni du radiamètre sous-marin portatif D.G.E.T.

plongeurs portent un sous-vêtement en coton pour éviter les effets de placage et assurer un certain confort thermique.

L'alimentation par air, en circuit ouvert, est assurée à partir de blocs bibouteilles équipés de détendeurs du type " Mistral " modifiés par la C.E.A. pour éviter toute entrée d'eau dans le circuit respiratoire par la soupape d'expiration (bec de canard).

Les liaisons plongeurs-surface sont assurées par téléphone à fil. La cagoule de l'habit est équipée d'un micro et d'un écouteur incorporés reliés par fil au poste de surface. Cet équipement permet de suivre les plongeurs de la 


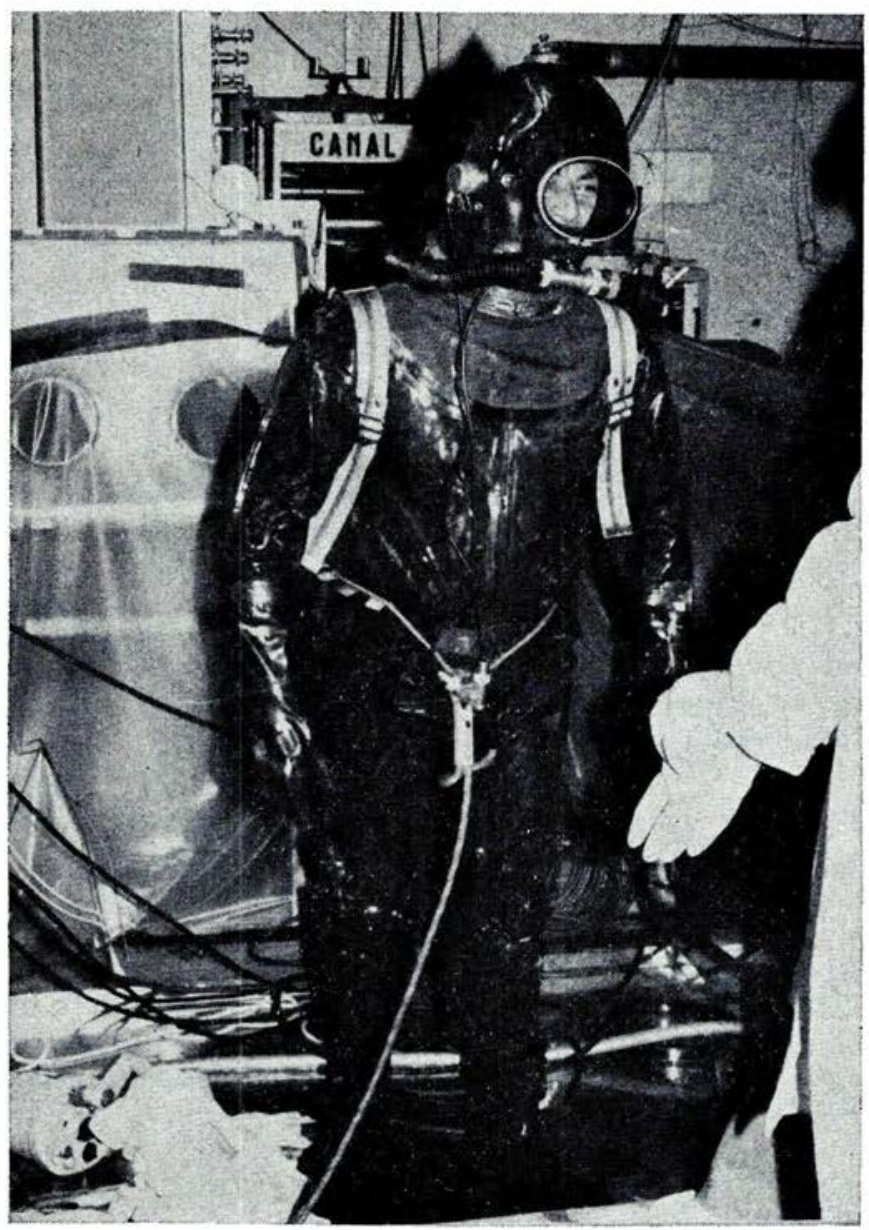

FIG. 2. - Plongeur équipé de la tenue étanche Draeger du type à "volume constant " avec téléphone à fil incorporé à la cagoule.

surface (aspect sécurité) et de diriger leur action en phonie, au vu des constatations faites au fond et des images reçues sur l'écran de télévision.

Le chantier de surface dispose, en outre, pour assurer la sécurité, d'équipements de plongée en réserve en cas d'intervention rapide de secours, d'une valise de réanimation respiratoire à l'oxygène de type AIROX et d'une pharmacie de premier secours.

Un détecteur I.P.A.B. (à double isolement) permet d'effectuer à la sonde le contrôle des plongeurs à leur sortie de l'eau (mesures possibles en alpha, bêta, gamma). Pour assurer, sous l'eau, la détection et la mesure de l'irra- 
diation gamma, les plongeurs disposent d'un radiamètre portatif de type D.G.E.T. Cet appareil, adapté à la plongée, possède une chambre d'ionisation enveloppée d'une protection plastique en matériau "équivalent tissu ». Les mesures sont exprimées en millirad par heure et rad par heure.

L'éclairage du chantier est assuré par deux circuits étanches indépendants : circuit de $24 \mathrm{~V}$ branché en surface sur le circuit secours assisté de l'installation; circuit $220 \mathrm{~V}$ pour les projecteurs étanches à iode de $1000 \mathrm{~W}$ dont la sécurité est assurée par double isolement, plus des transformateurs d'isolement sur chaque ligne.

Rappelons que la sécurité hydraulique du chantier est assurée.

\section{La surveillance médicale des plongeurs : le médecin du travail.}

Dès le début de l'étude de faisabilité, le médecin du travail est associé aux travaux. Plongeur lui-même, il bénéficie largement de sa connaissance des problèmes liés aux travaux sous-marins et de son intégration à l'équipe des plongeurs.

La surveillance médicale spéciale comporte deux aspects : aptitude médicale aux travaux sous-marins; aptitude médicale aux travaux sous rayonnements ionisants.

Du fait de leurs activités habituelles au C.E.A./Essais, les personnels concernés possèdent déjà cette double aptitude : plongeurs professionnels et agents de protection radiologique, ils subissent régulièrement les examens systématiques de surveillance médicale prévus par la réglementation. Par ailleurs, les examens de surveillance radiotoxicologique sont demandés par le médecin du travail du groupe au laboratoire spécialisé du service médical du travail du Centre de Saclay : anthroporadiamétrie : mesure de la radioactivité (naturelle ou due à une contamination éventuelle) du corps humain; contrôles urinaires : pour la détection et l'évaluation d'une éventuelle contamination par eau tritiée.

\subsection{SYNTHÈSE DE L'ÉTUDE DE POSTE}

L'étude de poste proprement dite est menée par le médecin du travail en coilaboration étroite avec le chef des plongeurs. Les problèmes concrets liés aux contraintes humaines sont analysés sous un double aspect :

- ergonomie pratique du futur chantier, planification de la prévention et des moyens de sécurité;

- mise en évidence des interventions possibles, au bénéfice de l'exploitant (feed-back, développement des possibilités de travaux de maintenance).

\section{Organisation du chantier sous l'angle de la prévention et de la sécurité}

Les plongées ont lieu sous faible profondeur $(7 \mathrm{~m})$ en espace clos assez exigu. Toutefois, les possibilités d'évolution sont suffisantes. Dans ces condi- 
tions, il n'est pas nécessaire d'utiliser un caisson de recompression. Par contre, il faut prévoir, en surface, un appareil de réanimation respiratoire à l'oxygène. En effet, le choix indispensable d'un vêtement du type à volume constant pose un problème particulier de sécurité propre à tous les équipements isolants : l'embout respiratoire solidaire de la cagoule interdit tout changement d'appareil au fond en cas (très improbable) de blocage du détendeur. Dans cette éventualité, le plongeur doit revenir en surface en panne d'air, enfermé dans son habit. Il reçoit, dans ce cas, l'assistance du deuxième plongeur pour effectuer un retour rapide (les distances sont réduites) avec le moins d'effort physique possible. Le poste d'oxygène en surface permet d'intervenir immédiatement sur une hypoxie.

La sécurité au fond obéit aux normes des travaux sous-marins : plongées par équipe de deux, liaisons plongeurs-surface par téléphone (et par télévision pour certaines séquences), éclairage des points de travail, moyens d'intervention rapide disponibles en surface (équipement de secours et plongeur de réserve).

Sous l'angle de la sécurité radiologique, le chantier de surface est organisé en zone contrôlée : balisage, accès réglementé, délimitation de circuits logiques chauds et froids pour les personnels, les équipements et les matériels de chantier (rôle du Service de Protection contre les rayonnements).

La phase d'assainissement radiologique nécessite l'emploi de matériels adaptés à l'utilisation au fond et au problème radiologique posé par le passage fond-surface : il s'agit, en effet, de maintenir les avantages de la protection par l'eau tout au long des manœuvres de recueil et d'évacuation des déchets actifs. Des fûts de 2001 remplis d'eau sont préparés pour recevoir en surface les filtres de la suceuse (granulométrie fine) ainsi que les conteneurs prévus pour les déchets de grosse granulométrie. Le problème de la manutention et de l'évacuation des déchets en surface se trouve ainsi ramené au transfert du fût de 2001 présentant une activité gamma pratiquement négligeable.

\subsection{EN CONCLUSION DE L'ÉTUdE DE POSTE ON ABOUTIT AUX PRÉVISIONS SUIVANTES :}

Les plongées doivent se dérouler sans contraintes inhabituelles, à raison de 2 heures de plongée en moyenne par jour et par agent sur une période de 1 mois environ. Les próvisions radiologiques sont les suivantes (doses intégrées) :

- de l'ordre de 50 à 100 mrem pour l'organisme entier dont environ 10 mrem en tritium;

- des équivalents de doses au poignet de quelques dizaines de millirems pour les opérations d'assainissement;

- contamination par les particules en suspension : ce risque doit être pratiquement négligeable.

vOL. $14-\mathrm{N}^{\circ} 2$ 


\section{DÉROULEMENT DES TRAVAUX : CONSTATATIONS FAITES SUR LE CHANTIER}

L'étude du chantier couvre deux périodes de 6 semaines environ, en 1976 et 1977 : les observations de 1976 ont été très exactement retrouvées l'année suivante. La préparation et l'équipement du chantier de surface d'une durée de 1 semaine environ correspond aux travaux habituels de mise en place des matériels. Aucune particularité n'est à noter. Le premier temps du chantier de plongées proprement dit, 2 jours environ, comprend l'ouverture et le verrouillage en position de sécurité des accès, la reconnaissance radiologique des capacités, l'ouverture et le blocage des grilles, la mise en place de l'éclairage d'ambiance du chantier ( $24 \mathrm{~V}$ secouru).

L'assainissement radiologique, qui prend 1 semaine environ, intervient avant le démarrage des interventions techniques. Sa durée est évidemment fonction de la dissémination, du volume et de l'activité des déchets. Pour ceux de grosse granulométrie, une à deux plongées suffisent. Saisis à la pince, ils sont déposés, sous eau, dans un conteneur placé à l'écart des points de passage habituels des plongeurs. Les dépôts actifs de faible granulométrie sont aspirés par suceuse à aspiration d'eau dans des filtres stockés sous eau et dont on limite l'activité à 1 rad au contact dans l'eau. Pour éviter les manutentions irradiantes en surface, ces filtres sont déposés en fûts de 2001 munis d'un support de centrage, et remplis d'eau, ce qui ramène l'activité en surface à $10-15 \mathrm{mrad} / \mathrm{h}$ au contact. Dans toutes ces opérations d'assainissement, l'effet de protection apporté par l'eau est maintenu. Les avantages d'une telle méthode sont considérables : les gestes sont simples, la protection des personnels excellente.

Les travaux techniques proprement dits se déroulent sur 5 semaines en moyenne : visite systématique des capacités, photographies après mise en place de projecteurs de $1000 \mathrm{~W}$, séquences télévisées enregistrées, changement de l'opercule d'une vanne, pose d'enduits et visite d'une gorge de batardeau au niveau de la piscine du réacteur.

Le déséquipement en fin de chantier porte sur 1 semaine et ne pose aucun problème particulier.

On remarque qu'un tel chantier, singulier à première vue, se déroule de manière parfaitement classique sans qu'apparaisse de séquence critique. Cette observation est d'ailleurs recoupée par l'analyse du déroulement des travaux chez les plongeurs dont certains points sont particulièrement significatifs :

L'équipement : les plongeurs le connaissent bien. Ils ont déjà largement utilisé le vêtement à volume constant. Sa seule particularité est son adaptation aux plongées en eau contaminée, ce qui n'entraîne pas de contrainte supplémentaire. Les palmes ne sont pas utilisées, il n'y a pas de longues distances à parcourir, et on limite ainsi la remise en suspension. Les plongeurs progressent facilement en se déhalant dans les canaux, ou en position " de cosmonaute " dans les capacités de grand volume. 
L'espace clos : sur ce chantier, il n'existe qu'une seule sortie. Les plongeurs assurent donc leur sécurité sur deux points, verrouillage systématique des portes et grilles en position ouverte, suivi permanent des plongeurs (en équipe de deux) par liaison phonique et, dans certaines séquences, par télévision en circuit fermé. Aucun sentiment d'insécurité n'est extériorisé.

La prévention radiologique leur apparaît particulièrement sûre. Les règles habituelles des travaux de l'industrie nucléaire sont appliquées, elles sont très familières aux plongeurs. Les équipements étanches leur assurent une protection efficace vis-à-vis d'une contamination éventuelle par remise en suspension, l'eau leur apporte une excellente protection vis-à-vis des rayonnements gamma.

La fatigue, analysée subjectivement par les plongeurs, leur apparaît équivalente à celle de travaux sous-marins classiques en volume constant. Cet équipement, il est vrai, diminue sensiblement l'aisance des mouvements malgré la souplesse du tissu caoutchouté utilisé (placage) mais dans une mesure très compatible avec la gamme des travaux à effectuer.

La température de l'eau est stabilisée à un niveau confortable. Par ailleurs, la fatigue inhérente au travail en pression est assez limitée en raison de la faible profondeur du chantier $(7 \mathrm{~m})$.

\section{Cas particulier de la plongée dans la piscine-réacteur}

Il s'agit d'une plongée à la verticale, en eau libre dans les mêmes conditions d'équipement et d'évolution que précédemment. Cependant, dans ce cas précis, la sécurité radiologique des plongeurs qui est conditionnée par l'épaisseur de l'écran-eau vis-à-vis des rayonnements gamma, est fonction de la distance cœur-plongeur. Une première approche radiologique permet de délimiter la zone à ne pas dépasser en utilisant un radiamètre portatif étanche D.G.E.T. Un balisage ou un repérage par rapport à la paroi piscine évite toute fausse manœuvre. Cette première approche radiologique réclame, bien entendu, une parfaite attention de l'opérateur.

Au cours des travaux et à la fin, la même impression est exprimée, sans discordance, par tous les plongeurs du groupe. Il se traduit par la sensation d'un chantier parfaitement normal et de conditions de travail connues et bien maîtrisées. Ce comportement contribue à donner au chantier lui-même une ambiance méthodique et détendue. Il faut aussi reconnaître que l'intérêt de tels travaux, réalisés pour la première fois sur une pile piscine, entraîne une motivation élevée chez tous les participants.

\section{ANALYSE CRITIQUE DE L'ÉTUDE ERGONOMIQUE}

Comme nous l'avons vu précédemment, l'étude ergonomique menée au niveau de la conception correspond très exactement aux données objectives de l'étude de poste menée sur le chantier, ainsi qu'aux données subjectives 


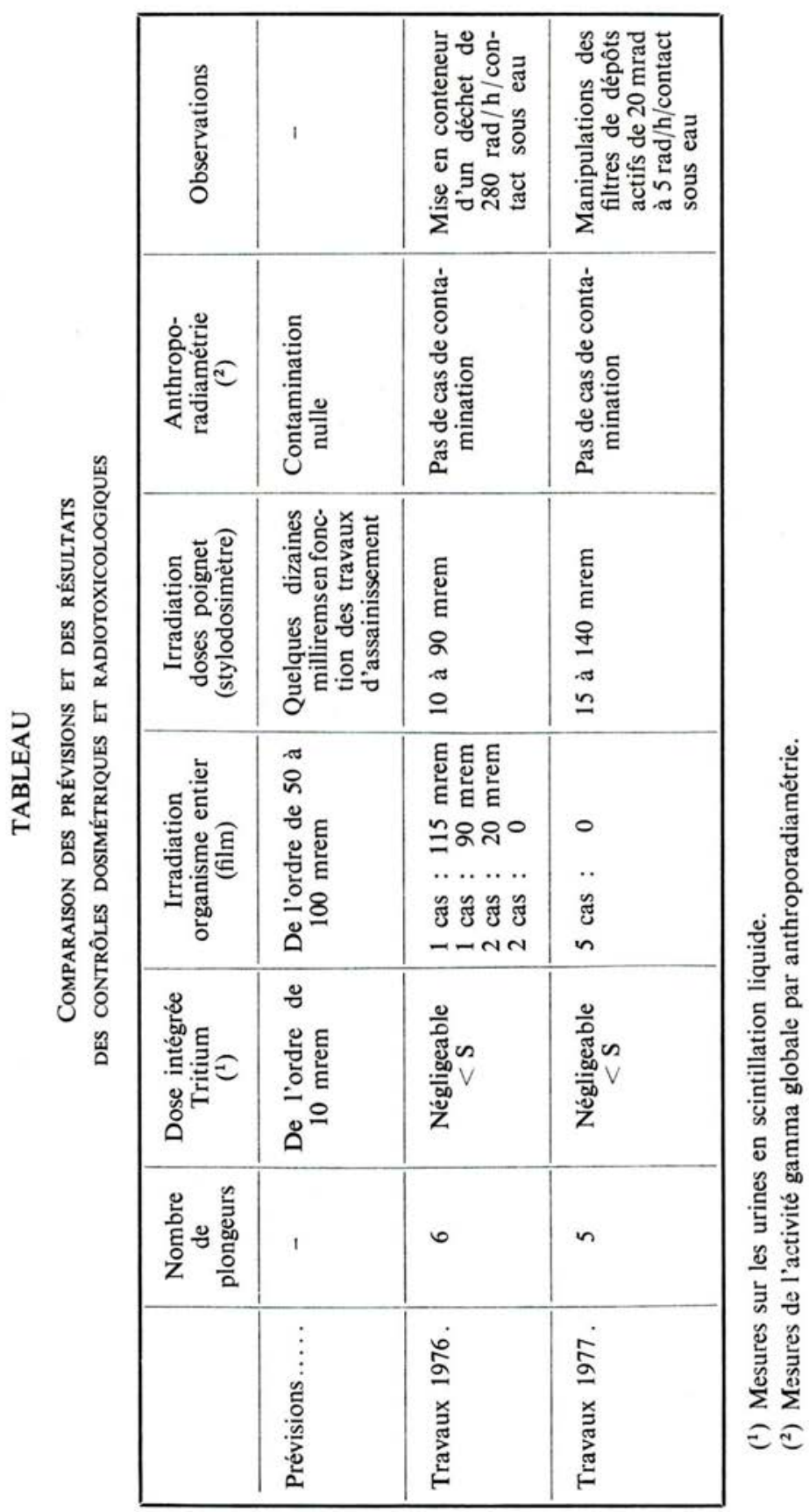


exprimées par les participants. Il en est de même pour les données chiffrées de la dosimétrie individuelle et des contrôles radiotoxicologiques des plongeurs (tableau). On peut donc admettre qu'au niveau de la conception, l'étude multidisciplinaire a intégré de façon très complète les paramètres humains et techniques du chantier, conclusion confirmée par les deux périodes de travaux menés en 1976 et en 1977.

\section{CONCLUSIONS}

Nous nous sommes attachés à exposer les différentes phases de développement d'une étude ergonomique multidisciplinaire axée sur la prévention et les conditions de travail d'un chantier nucléaire de type nouveau, analysé globalement dès le niveau de la conception. L'intérêt d'une telle démarche, classique en ergonomie et en médecine du travail, réside en partie dans l'aspect inhabituel des travaux, mais surtout, à notre avis, dans les bénéfices qui en sont retirés en matière de sécurité et de contraintes de travail.

La coopération étroite, méthodique de tous les participants a permis de dégager des solutions aisées à mettre en œuvre sur le terrain. Dans ce travail d'équipe, le noyau chef des plongeurs/médecin du travail a joué un rôle de catalyseur. Leurs connaissances des problèmes pratiques liés à ce type de chantier a permis d'intégrer, dès la phase de la conception et tout au long des travaux, les données concernant la prévention et les conditions de travail. Par feed-back, les ingénieurs de l'installation se sont vu ouvrir de nouvelles possibilités d'interventions techniques. Du début de l'étude préalable à la fin de la deuxième campagne de travaux, nous sommes passés du concept de chantier insolite à celui de chantier industriel réglé, peu contraignant et sûr, sous le double aspect technique et humain.

Pour le médecin du travail, une telle démarche ergonomique n'a rien de singulier, elle est de pratique courante dans le domaine des travaux en environnement hostile. Son intérêt, et il n'est pas mince, réside surtout dans sa simplicité et dans l'aide apportée en pratique à l'homme au travail qu'il soit responsable ou exécutant. Pour l'ingénieur, de nouvelles perspectives sont ouvertes en ce qui concerne les opérations de vérification et de maintenance des réacteurs-piscine. Au plan de la méthode de travail proprement dite, l'intervention par plongeurs n'est que l'application à l'homme du principe de manutention sous écran d'eau de matériels actifs. Soigneusement préparée, elle permet, comme nous l'avons vu, de réaliser, en toute sécurité, de substantielles économies en temps et en moyens. Cette technique d'intervention pourrait aussi être extrapolée à d'autres installations et ouvrir ainsi de nouvelles possibilités d'actions dans l'industrie nucléaire. 